\title{
La difesa nel caso Cogne
}

\author{
Elena Bianchini ${ }^{\bullet}$
}

\section{Riassunto}

L'Avv. Paola Savio, nell'intervista effettuata presso il suo studio a Torino, avanza alcune riflessioni in riferimento al "caso Cogne", in particolare relativamente all'ultima fase del percorso giudiziario che ha portato all'ordinanza emessa dal Tribunale di Sorveglianza di Bologna il 26 giugno 2014. L'avvocato sottolinea la sinergia e 1'“incontro di professionalità" avvenuto tra il CTU, il Prof. Augusto Balloni, e il consulente di parte, il Prof. Pietro Pietrini, la bontà dei supporti forniti alla sua assistita in fase rieducativa e auspica, come già pubblicamente dichiarato, che la famiglia $\mathrm{F}$. possa essere dimenticata e cadere nell'oblio.

\section{Résumé}

Au cours de l'entretien qui a eu lieu dans son cabinet à Turin, Maître Paola Savio formule quelques considérations concernant «l'affaire Cogne ». Une attention particulière est accordée à la dernière phase du parcours judiciaire où le Tribunal de l'application des peines (Tribunale di Sorveglianza) de Bologne a ordonné, le 26 Juin 2014, la mise en détention à domicile spéciale d'AMF.

Maître Savio souligne l'importance de la synergie et de «l'échange de compétences » entre professionnels (l'expert indépendant, Prof. Augusto Balloni, et celui de la défense, Prof. Pietro Pietrini), ainsi que de la qualité de l'aide apportée à sa cliente pendant la phase de réhabilitation en prison.

Enfin, elle espère, comme cela a déjà été déclaré publiquement, que la famille F. puisse tomber dans l'oubli.

\section{Abstract}

Lawyer, Paola Savio, during the interview carried out at her law firm in Turin, makes some considerations about the so-called "Cogne case". Particular attention is paid on the final step of the judicial action where the Surveillance Court of Bologna made a special home detention order on 26th June 2014.

The lawyer underlines the importance of the synergy and the successful meeting among professionals (the independent technical consultant, Prof. Augusto Balloni, and the defence consultant, prof. Pietro Pietrini). She is also pleased with the quality of the help given to her client during the rehabilitation phase.

Finally, she hopes, as already publicly stated, that the family F. would no longer be in the focus of attention.

Key words: Cogne case; defence lawyer; judicial action; rehabilitation of offenders; mass media.

\section{Introduzione.}

Venerdì 6 febbraio 2015 mi sono recata a Torino per intervistare l'Avv. Paola Savio, difensore di AMF. L'avvocato si è dimostrata molto disponibile e aperta al dialogo e si è posta nei confronti dell'intervista con una sincerità ed una onestà non scontate e per questo la ringrazio molto.

I temi che sono stati affrontati nel corso dell'intervista hanno riguardato la consulenza tecnica d'ufficio e gli accertamenti effettuati dal consulente tecnico d'ufficio, nominato dal Tribunale di Sorveglianza di Bologna, il Prof. Balloni, e dal consulente tecnico di parte, il Prof. Pietrini, nonché il successivo approfondimento richiesto dal Tribunale. L'avvocato sottolinea come "la perizia da ultima espletata abbia in qualche modo ricompreso tutte quelle tappe importanti che una perizia di questa natura deve ripercorrere e che il supplemento di perizia è stata una decisione giusta e molto responsabile da parte del Tribunale".

\footnotetext{
- Dottore di ricerca in sociologia, assegnista di ricerca presso il C.I.R.Vi.S. (Centro Interdisciplinare di Ricerca sulla Vittimologia e sulla Sicurezza), Dipartimento di Sociologia e Diritto dell'Economia, Università di Bologna.
} 
Grande rilevanza viene inoltre data all'interferenza dei mass media, in fase processuale e soprattutto nel percorso rieducativo, vissuta come assolutamente devastante e anche controproducente: "anzi io sono fermamente convinta che se non ci fosse stato un accanimento mediatico di questo tipo e ci fosse stata più sobrietà e tranquillità nelle fasi processuali che hanno contraddistinto tutto il processo, forse alcune cose sarebbero andate in modo diverso". E' proprio per questa motivazione che l'avvocato invoca il diritto all'oblio per la famiglia ed auspica che l'intera vicenda processuale venga dimenticata.

Il percorso rieducativo dell'assistita, altro argomento oggetto dell'intervista, viene giudicato assai positivamente: "io credo che la strada che è stata scelta dal Tribunale sia una strada perfetta, in quanto la psicoterapia si è andata ad aggiungere ad una rete che era già in atto", rete composta da interventi posti in essere dai servizi territoriali e dagli aiuti familiari e amicali.

Infine, non sono stati trascurati gli aspetti emotivi personali dell'avvocato, che inevitabilmente sono presenti in una vicenda processuale così peculiare e che si è protratta temporalmente.

\section{L'intervista'}

Domanda: Ha ricevuto il materiale che le è stato inviato riguardante il caso di AMF?

Risposta: Sì

D: Le sembra una sintesi adeguata per una valutazione scientifica del caso?

R: Sì, non ho assolutamente nulla da osservare. Mi è parso che il materiale, gli interventi di questi Autori abbiano un focus delimitato e cioè focalizzino più l'attenzione sulla personalità di AM vista come AM detenuta, sull'esperienza della carcerazione e quindi c'è un po' tutto, anzi, credo che non manchi nulla dal punto di vista dell'accertamento da ultimo intervenuto.

D: Gli accertamenti effettuati, anche alla luce dell'ordinanza del Tribunale di Sorveglianza, le sembrano essere stati sufficienti?

R: Io credo che questo processo abbia implicato una cultura dell'approfondimento psichiatrico a partire dalle indagini preliminari per giungere alla fase esecutiva "finale". Questo processo è sicuramente stata un'esperienza molto importante anche per il non addetto ai lavori, per il non psichiatra, il non tecnico. Questo per dire, sulla base dell'esperienza maturata, che io credo che la perizia da ultima espletata abbia in qualche modo ricompreso tutte quelle tappe importanti che una perizia di questa natura deve ripercorrere. Quindi, forse, sarebbe meglio rivolgere ad uno psichiatra forense una domanda di questo tipo, non ad un avvocato, ma essendo io un avvocato che ha avuto un'esperienza specifica proprio in questo caso, $\mathrm{mi}$ viene da dire che il perito non abbia tralasciato assolutamente nulla. Mi sembra che ci sia stata proprio una verifica a trecentosessanta gradi, che comprende la natura dei test somministrati, le modalità e, in particolare, il colloquio clinico, questo grande e importante passaggio che consiste in un approfondimento sulla psiche, sulla mente di una persona che invece in altri casi, ad esempio, è totalmente mancato.

D: Come giudica il fatto che i periti abbiano incontrato la famiglia assieme alla periziata?

R: Questo approccio mi è piaciuto molto, queste scelte mi hanno tranquillizzata.

1 L'intervista è stata realizzata nello studio privato 


\section{D: Nell'ambito della consulenza d'ufficio, dal} punto di vista dell'avvocato difensore, quali erano gli elementi che più la preoccupavano: la documentazione psichiatrica passata, l'analisi del contenuto della documentazione acquisita, l'esito dei colloqui, i risultati dei test mentali?

R: Io penso che tutto spaventi e nulla debba spaventare, in quanto sulla base di questa esperienza specifica, cioè il caso Cogne, ho notato come la testistica debba necessariamente fornire il suo contributo, ma che comunque sia il colloquio clinico a dover fare da faro. Quando ci è stato spiegato che saremmo partiti dal colloquio clinico, io mi sono tranquillizzata, ma si è tranquillizzata anche AM. Io qui non ho temuto nulla, nel senso che era abbastanza chiara la strada che i periti avrebbero percorso e non avevo motivo di temere nulla.

\section{D: Quindi è stato importante l'aspetto della} fiducia, della serietà, della competenza?

R: E' fondamentale. Ci sono situazioni che vengono proprio complicate dal tipo di atteggiamento tenuto dal professionista. In casi come questi si rischia di pregiudicare la prova o l'accertamento. In questo caso è fondamentale trovare l'empatia, trovarsi d'accordo, essere soprattutto chiari e onesti e dire quello che si pensa nel modo educato che tutti ci aspettiamo. E non è così scontato: io credo che in questo caso ci sia stato davvero un incontro di professionalità tra perito e consulente di parte, perché si sono subito fidati l'uno dell'altro e quindi sono arrivati ad avere, secondo me, un prodotto un po' sui generis, che non si è soliti avere.

D: Questo può avere giovato per una decisione serena del Tribunale?

dell'avvocato e deregistrata dal supporto digitale.
R: Sì, ma non solo in questo senso. Il Tribunale sicuramente avrà recepito questa sintonia che, a volte, può anche essere fraintesa. Nel rispetto dei reciproci ruoli, per me si ha sintonia quando una persona parla chiaro ad un'altra, quando non ha un doppio fine, quando c'è rispetto. Per me sintonia non significa mettersi d'accordo sull'esito finale, sintonia vuol dire: bene, c'è una testistica che uno propone e l'altro professionista esprime una propria valutazione, proponendo di aggiungere, ad esempio, un altro test. Ecco in questo caso questa sintonia c'è stata, ed ha giovato a tutti eccome. Ho letto i contributi ed effettivamente c'è un passaggio che mi è piaciuto molto, perché si sottolinea proprio l'importanza che questo approfondimento ha avuto per tutti: dalla diretta interessata, ai tecnici che sono stati incaricati, al Tribunale.

D: Il supplemento di perizia come è stato valutato nella prospettiva dell'avvocato difensore? Come un rischio o come un necessario approfondimento?

R: E' stata una decisione secondo me giusta e molto responsabile del Tribunale. Ovviamente, in questo caso giocava un ruolo fondamentale anche l'attenzione mediatica che c'è stata per così tanto tempo. Ed era giusto per la serenità di tutti, secondo me, fare un approfondimento in più piuttosto che farne uno in meno. Prima mi aveva chiesto quali erano gli elementi che più mi preoccupavano: non era una vera e propria preoccupazione, ero scettica sull'approfondimento grafologico, per esempio, perché non ne conoscevo i contenuti, era uno strumento a me ignoto. Però, fidandosi del perito che lo decide, esso diventa poi accettabile: d'accordo con tutti ovviamente, con AM, con il Prof. Pietrini, ci 
siamo fidati del perito. Quindi l'approfondimento è stato molto importante, come dicevo prima, e secondo me ha permesso, tra le altre cose, di passare subito all'aspetto concreto e pratico, cioè l'approfondimento mirava ad individuare una strada di appoggio psicologico per il passaggio alla libertà, chiamiamolo così, e questo è stato un passaggio che secondo me è stato chiarito sin dall'inizio dal Prof. Balloni e segnalato come necessario. È stata vissuta sicuramente come un segnale di fiducia da raccogliere e così si è fatto. Tra l'altro io non so in quante altre situazioni si possano verificare o si siano verificati in passato dei percorsi concreti e positivi come in questo caso. Devo però dire che qui, come ha giustamente osservato lo stesso Prof. Balloni, la perizia va un po' oltre quelli che sono i contenuti tipici di una perizia psichiatrica in ambito forense, ma ha un contributo criminologico e secondo me psicologico di supporto. Io l'ho intesa così, non me ne sono resa conto subito, ma alla fine quello che mi è venuto da pensare è stato: il clinico, oltre che lo psichiatra forense, ha iniziato a mettere in atto già un percorso, non ce ne siamo accorti subito, adesso che stiamo meglio, ce ne stiamo accorgendo e funziona!

D: Le interferenze dei mass media come le ha valutate e come sono state percepite dalla sua assistita? A suo avviso sarebbe opportuno mantenere una maggiore segretezza per quanto riguarda i mass media o lasciarli entrare più approfonditamente nell'ambito dei percorsi giudiziari?

R: Devastante. Anzi io sono fermamente convinta che se non ci fosse stato un accanimento mediatico di questo tipo e ci fosse stata più sobrietà e tranquillità nelle fasi processuali che hanno contraddistinto tutto il processo, forse alcune cose sarebbero andate in modo diverso. Sono ancora adesso abbastanza colpita e ogni volta non riesco ad abituarmi: quando c'è stata l'udienza in Tribunale di Sorveglianza di Bologna e mi sono ritrovata, come anni fa, i gomiti addosso, ho detto 'ci risiamo'. Incredibile. Ma che cosa produce tutto questo? Non si tratta solo del livello di informazione, ma viene detta una cosa piuttosto che un'altra, non è il riportare cose che secondo me sarebbe opportuno fossero riferite soltanto dai diretti interessati o dall'autorità giudiziaria, ma io credo che abbia un altro risvolto, e cioè la capacità-incapacità della persona che riceve tutta questa attenzione di affrontarla. $\mathrm{Si}$ possono innestare dei comportamenti diversi da quelli che la persona stessa avrebbe magari tenuto naturalmente e che possono poi essere fraintesi. Noi qui ancora oggi, a distanza di anni dalla sentenza di condanna, ci dobbiamo confrontare con quel maledetto filmato in cui AM dice "Ho pianto troppo?" e ancora si attribuiscono ad esso mille interpretazioni laddove ce n'è solo ed esclusivamente una e guarda caso in questa perizia gli esperti erano assolutamente d'accordo sull'interpretazione da dare a quella frase, perché dopo averla conosciuta, averla incontrata otto, nove, dieci volte, ecco che diventa chiaro a tutti qual è il contenuto di quella frase. E invece no, siamo ancora lì a dover parlare di "Ho pianto troppo". Ecco, questi sono purtroppo dei risvolti che hanno avuto un effetto devastante anche proprio sull'apparato argomentativo della sentenza di condanna. Una persona deve poi collocare "Ho pianto troppo": è la prima intervista che fa $\mathrm{AM}$, donna disfatta dalla tragedia della perdita del figlio, donna che si disfa 
sempre di più perché il cerchio si stringe e si sta stringendo, quella è un'intervista che avviene pochi giorni prima del suo arresto, e altre persone, altri suoi familiari decidono al posto suo che doveva difendersi pubblicamente; poi stavano puntando il dito verso di lei, come naturale che avvenisse, che la Procura facesse. Lo stesso Prof. Balloni nella sua perizia parla proprio di questa modalità di parlare lamentoso, cantilenante di AM perché quando piange è monocorde, non si si capisce che cosa dice: allora se uno innesta tutte queste informazioni, la frase "Ho pianto troppo" cambia completamente. Ecco, l'esempio è questo, devastazione.

D: Quali sono secondo lei gli aspetti più temibili nell'ambito delle interferenze dei media con riferimento al progetto rieducativo?

R: Noi lo abbiamo chiesto, e lo ha chiesto anche il Prof. Balloni, pubblicamente, che venga dimenticata. Oramai al giorno d'oggi, nell'epoca di internet, il diritto all'oblio non c'è più, non esiste più. Devo però dire che il fatto di trovare il giornalista appollaiato sull'albero per vedere quanti panni stende, se li stende, se c'è il sole o piove, se esce, non esce, io credo che questo sia proprio un perseguitare una persona, una famiglia che avrebbe bisogno di essere lasciata tranquilla. Non si tratta più di interesse alla notizia, all'avvenimento storico, cioè la condanna di AM per l'omicidio del figlio o la scarcerazione, ma del volere a tutti i costi un suo sguardo, una sua frase, una dichiarazione sua o di chi le sta vicino per questa fame o sete di sfumature della sua vita che non interessano a nessuno, solo ai giornalisti che titolano, perché a me non viene in mente a chi possa interessare $\mathrm{i}$ panni che stende, o se abbraccia amorevolmente il marito o il figlio.
Allora si tratta di morbosità, alimentata all'inizio dalla stessa famiglia. C'è stata proprio un'esasperazione delle sensazioni, dei sentimenti di tutti che hanno portato a sbagliare, e questi sbagli poi sono cresciuti in maniera esponenziale. Si tratta di un mondo completamente sconosciuto e insidioso, molto insidioso.

\section{D: Nei casi di figlicidio, nel percorso} rieducativo, quali supporti dovrebbero essere forniti alle madri che si macchiano di tale delitto?

R: Io credo che per AM la strada che è stata scelta dal Tribunale sia una strada perfetta. Perché non si tratta solo di psicoterapia, ma la psicoterapia si è andata ad aggiungere ad una rete che era già in atto. Pensiamo al lavoro: c'è un'attività lavorativa coltivata e che funziona, perché sono tutti contenti di AM, è una brava, produce; poi c'è il programma dei servizi territoriali che seguono la famiglia e soprattutto hanno sempre seguito i minori, sono stati molto presenti e molto attenti, quindi la psicoterapia ha completato un quadro molto ricco di sfaccettature, che poi portano tutte alla normalità, perché è una donna che torna a lavorare, che torna ad occuparsi della famiglia, della scuola e che quindi si preoccupa di come comportarsi con loro, perché, dopo anni di carcerazione, una persona può chiedersi 'che cosa dirò', 'come mi comporterò' ?. Quindi no, non credo che manchi nulla, l'aiuto viene da varie parti. C'è poi questo marito, questa famiglia, questo marito fedele, coerente, presente, che è diventato padre, è diventato madre, si è occupato di tutto; oltre a lui, ci sono i genitori di lui, adesso purtroppo suo papà non c'è più. Sono persone che appartengono a categorie diverse. 


\section{D: L'attenzione professionale viene influenzata} in questi casi da aspetti emotivi?

R: Ha dei lati positivi e negativi. L'indifferenza non può esserci in casi come questi. Ha sicuramente dei lati positivi, e lo dico adesso perché c'è molta più confidenza, e la confidenza, nel momento in cui uno si fida dell'altro, permette di parlare in modo diverso, senza il filtro del formalismo e questo secondo me permette di arrivare prima ad avere informazioni che servono o comunque a conoscere meglio la situazione. L'aspetto negativo può essere la produzione di emozione, ciò che noi non possiamo permetterci di avere nel momento in cui siamo chiamati a svolgere il nostro ruolo. Anche questo però non è sempre vero, perché anche l'avvocato ha un cuore, un'anima, e anche l'avvocato deve, secondo me, poter vivere la 'sua' vicenda come meglio crede. Quindi 1' emotività, in questo caso, è stata assai intensa per me.

D: Non si rischia di vivere le decisioni, anche quelle del Tribunale, in maniera personale?

R: No, no, quello mai. Nel senso che l'emotività gioca su di te, non gioca sul tuo assistito, quindi bisogna distinguere i piani, innanzitutto. Prima si è avvocati, poi si è affezionati alla parte che assisti e quindi ci si deve preparare, come per chiunque altro, alle questioni processuali, gli aspetti emotivi non ti devono disorientare. Ovviamente nel momento in cui, dopo un livello di guardia elevatissimo per tutto il tempo del processo, viene letta la sentenza, abbiamo il sacrosanto diritto di ridere, di piangere. E' giusto non reprimere, come si fa? E a chi dice che non prova nulla, a questi io non credo. Io non mi vergogno affatto di dire che quando sono tornata a casa la sera della sentenza della Corte d'Assise d'Appello, dopo aver sperato nell'assoluzione, perché c'erano dei vuoti, dei buchi neri nel ragionamento probatorio; dopo essere stata travolta dalla marea umana di giornalisti fuori dal Tribunale; dopo essere intervenuta in collegamento la sera, quando sono arrivata a casa, finalmente, sono scoppiata a piangere. Ho diritto anch' io a versare due lacrimucce per quella tensione estenuante, ma non me ne vergogno. Come è stata dura dire ad AM che la Cassazione aveva rigettato il ricorso, però questo ti porta ad un' unione incredibile con la persona che oramai conosci più di molti tuoi amici.

D: Il caso in esame le ha fatto pensare alla scellerata Medea, soprattutto in rapporto a quanto scriveva Euripide: "Come dunque la città dai sacri fiumi, e la terra che ospita gli amici, accoglierà te infanticida, fra tutti la più empia?"

R: Mai, neanche per un secondo, assolutamente no. Ho visto la tragedia a Siracusa, nel teatro più bello che c'è in Italia secondo me, lì è paradisiaco. Ero con un gruppo di lavoro a Siracusa, un gruppo di varie professionalità per approfondire il tema della prova scientifica nel processo. Una sera siamo andati a vederla, anche con alcuni protagonisti della vicenda Cogne, proprio con persone che in qualche modo hanno preso parte a questa vicenda. Medea aveva, come dire, una situazione completamente diversa, mentre AM non ha mai avuto quella situazione, neanche per un secondo. Io sono fermamente convinta della sua innocenza e lo sarò sempre.

\section{Conclusioni.}

L'intervista all'Avv. Paola Savio ha sottolineato diversi aspetti riguardanti l'ultima vicenda 
processuale del cosiddetto "caso Cogne", cioè la decisione del Tribunale di Sorveglianza di Bologna di concedere ad AMF la detenzione domiciliare speciale. Giova ricordare che, con l'emanazione della Costituzione italiana, entrata in vigore nel 1948, la rieducazione e l'umanizzazione della pena vengono sancite dall'art. 27 comma $3^{\circ}$, il quale stabilisce che: "Le pene non possono consistere in trattamenti contrari al senso di umanità e devono tendere alla rieducazione del condannato". Dal principio rieducativo della pena, che è dunque un dettame costituzionale, e che ritrova nella Legge 26 luglio 1975, n. 354 denominata: "Norme sull'ordinamento penitenziario e sull'esecuzione delle misure privative e limitative della libertà" la sua prima e organica attuazione a livello di legislazione ordinaria, scaturiscono differenti corollari inerenti, in particolare, alle misure alternative alla detenzione, ai permessi, ai programmi di trattamento: la pena inflitta con sentenza di condanna si può modificare e, nel corso della sua esecuzione, può essere attenuata dal punto di vista qualitativo e quantitativo. E' dunque di fondamentale importanza valorizzare il reinserimento sociale del reo e riconoscere l'autore di un reato quale persona titolare di diritti che permangono anche nei confronti dell'amministrazione penitenziaria ${ }^{2}$.

La perizia psichiatrica e il ruolo svolto dai periti assume una rilevante importanza e, nella vicenda in esame, gli accertamenti peritali vengono considerati dall'avvocato esaurienti e soddisfacenti: "mi viene da dire il perito non ha tralasciato assolutamente nulla. Mi sembra che ci

2 Della Casa F., La magistratura di sorveglianza: organizzazione, competenze, procedure, G. Giappichelli, Torino, 1994. sia stata proprio questa verifica a trecentosessanta gradi quindi la natura dei test somministrati, le modalità, l'importanza che hanno dato al colloquio clinico, questo grande $e$ importante passaggio di un approfondimento sulla psiche, sulla mente di una persona che invece in altri casi, ad esempio, è totalmente mancato"; in particolar modo "mi è piaciuto molto, devo dire, mi ha colpito sin da subito" il fatto che in un'occasione, i periti abbiano incontrato, assieme alla perizianda, anche la sua famiglia. Particolarmente importante è stato dare centralità al colloquio clinico e ai diversi incontri, circa dodici, che i periti hanno effettuato con AMF: "è il colloquio clinico a dover fare da faro. Quando ci è stato spiegato che si sarebbe partiti dal colloquio clinico, io mi sono tranquillizzata, ma si ̀̀ tranquillizzata anche AM'. Proprio in riferimento all'ambito peritale, l'avvocato ha sottolineato più volte 1'“incontro di professionalità tra perito e consulente di parte, perché si sono subito fidati l'uno dell'altro e quindi sono arrivati ad avere, secondo me, un prodotto un po' sui generis, che non si è soliti avere”. E' stata fondamentale la sintonia e l'empatia che è nata fra il CTU e il perito di parte, "sintonia che poi, a volte, può anche essere fraintesa. Per me sintonia non significa mettersi d'accordo sull'esito finale, sintonia vuol dire: bene, c'è una testistica che uno propone e l'altro professionista esprime una propria valutazione, proponendo di aggiungere, ad esempio, un altro test. Per me sintonia ̀̀ quando una persona parla chiaramente ad un'altra, non ha un doppio fine".

L'approfondimento di perizia, disposto dal Tribunale di Sorveglianza di Bologna, secondo l'intervistata "ha permesso, tra le altre cose, di 
passare subito all'aspetto concreto e pratico, cioè l'approfondimento mirava, si è poi visto, ad individuare una strada di appoggio psicologico per il passaggio alla libertă" e tale percorso è stato recepito molto positivamente anche dalla perizianda: "se mi indicano quella strada facciamola subito perché vuol dire che se non meritassi nulla non mi avrebbero segnalato nulla. $\grave{E}$ stata vissuta sicuramente come un segnale di fiducia da accogliere e così si è fatto. Tra l'altro io non so in quante altre situazioni si possano verificare o si siano verificati in passato dei percorsi concreti e positivi come in questo caso".

In una vicenda così esposta da un punto di vista mediatico, le interferenze dei mass media hanno avuto un effetto devastante. L'avvocato, infatti, afferma di essere fermamente convinta che se non ci fosse stato un accanimento mediatico di questo tipo e ci fosse stata più sobrietà e tranquillità nelle fasi processuali che hanno contraddistinto tutto il processo, forse alcune cose sarebbero andate in modo diverso. Infatti, "non si tratta solo del livello di informazione, ma viene detta una cosa piuttosto che un'altra, non è il riportare cose che secondo me sarebbe opportuno fossero riferite soltanto dai diretti interessati o dall'autorità giudiziaria, ma io credo che abbia un altro risvolto, e cioè la capacità-incapacità della persona che riceve tutta questa attenzione di affrontarla. Ecco che allora si possono innestare dei comportamenti diversi da quelli che la persona stessa avrebbe magari tenuto naturalmente e che possono poi essere fraintesi. [...] “.

Come infatti ben ricorda David Garland, "oggi, anche se le sanzioni penali non sono quasi più eseguite pubblicamente, esistono ancora prassi penali rivolte - e dunque comunicate - alla comunità sociale. Si pensi, in particolare, al dispositivo della sentenza e a ogni ulteriore commento che il giudice decide di aggiungere a questo importantissimo atto discorsivo: la pronuncia (o l'atto performativo) riguarda sicuramente il reo, ma anche le vittime, i potenziali delinquenti e il pubblico nella sua totalità, e ciò grazie alla diffusione dei media e allo spazio riservato agli spettatori nella stessa aula del processo" ${ }^{\text {. }}$. Se la pena ha dunque l'obbligo di reinserire socialmente il reo, una sentenza di condanna deve sicuramente cercare di contenere al minimo le "conseguenze sociali" negative che inevitabilmente si vengono a creare. Ecco che in questo quadro il processo rieducativo assume un ruolo centrale: la psicoterapia, indicata dal Tribunale come condizione imprescindibile per un corretto reinserimento sociale, " $h a$ completato un quadro molto ricco di sfaccettature, che se vogliamo poi portano tutte alla normalità, perché è una donna che torna a lavorare, che torna ad occuparsi della famiglia, della scuola e che quindi si preoccupa di sapersi comportare con loro". Infatti, ai fini di una corretta opera di risocializzazione, bisogna considerare la persona come un "complesso strutturato di tratti psicologici e comportamentali che dall'interazione col contesto ambientale trae ampi spazi di possibilità, che sono alla base della sua originalità e individualità’, ${ }^{\circ}$ in questo caso è rappresentata da una psicoterapia di supporto, dal coinvolgimento degli enti territoriali e da una

\footnotetext{
${ }^{3}$ Garland D., Pena e società moderna, Il saggiatore, Milano, 1999, p. 297.

4 Bisi R., "Giustizia, carcere, riabilitazione del criminale e solidarietà sociale, in Balloni A., Bisi R., Sette R. (a cura di), Manuale di criminologia - II.
} 
forte rete familiare, in particolar modo dal marito e dagli amici. Per quanto riguarda la famiglia, "noi lo abbiamo chiesto, e lo ha chiesto anche il Prof. Balloni, pubblicamente, che venga dimenticata".

Si è voluto infine indagare sugli aspetti emotivi e se questi possano influenzare la competenza di un professionista: infatti “l'indifferenza non può esserci in casi come questi'. L'avvocato sottolinea come ci siano degli aspetti sia positivi che negativi: "Ha sicuramente dei lati positivi, e lo dico adesso perché c'è molta più confidenza, e la confidenza, nel momento in cui uno si fida dell'altro, permette di parlare in modo diverso, senza il filtro del formalismo e questo secondo me permette di arrivare prima ad avere informazioni che servono o comunque a conoscere meglio la situazione. L'aspetto negativo può essere la produzione di emozione, ciò che noi non possiamo permetterci di avere nel momento in cui siamo chiamati a svolgere il nostro ruolo. Anche questo però non è sempre vero, perché anche l'avvocato ha un cuore, un'anima e anche l'avvocato deve, secondo me, poter vivere la 'sua' vicenda come meglio crede. Quindi l'emotività, in questo caso, è stata assai intensa per me".

\section{Bibliografia.}

- Balloni A., Criminologia e psicopatologia. Analisi di 110 perizie psichiatriche, Pàtron, Bologna, 2004.

- Balloni A., Bisi R., Sette R. (a cura di), Manuale di criminologia - I. Le teorie, Clueb, Bologna, 2013.

- Balloni A., Bisi R., Sette R. (a cura di), Manuale di criminologia - II. Criminalità, controllo, sicurezza, Clueb, Bologna, 2013.

- Bianchini E., La riabilitazione tra estinzione del reato e riparazione alla vittima. La sua applicazione in Italia $e$ in Belgio, Edizioni Accademiche Italiane, Saarbrüken, 2013.

- Bianchini E., "Il trattamento penitenziario del condannato", in Balloni A., Bisi R. Sette R., Manuale di criminologia, II. Criminalità, controllo, sicurezza, Clueb, Bologna, 2013, pp. 283-301.

- Bisi R., "Giustizia, carcere, riabilitazione del criminale e solidarietà sociale", in Balloni A., Bisi R., Sette R. (a cura di), Manuale di criminologia - II. Criminalità, controllo, sicurezza, Clueb, Bologna, 2013, pp. 273-281.

- Della Casa F., La magistratura di sorveglianza: organizzazione, competenze, procedure, G. Giappichelli, Torino, 1994.

- Garland D., Pena e società moderna, Il saggiatore, Milano, 1999.

Criminalità, controllo, sicurezza, Clueb, Bologna, 2013, p. 277. 